\title{
Challenges in the Histopathologic Diagnosis of Histiocytic Neoplasms
}

Karen L. Rech, MD, ${ }^{1}$ and Rong $\mathrm{He}, \mathrm{MD}^{1}$

\begin{abstract}
Histiocytic neoplasms, including Langerhans cell histiocytosis (LCH), Erdheim-Chester disease (ECD), and Rosai-Dorfman disease (RDD), present a diagnostic challenge due to nonspecific fibroinflammatory infiltrates and a diverse clinical presentation. The pathologist can play a key role in classification of these disorders through multidisciplinary collaboration and correlation of pathologic features with clinical and radiologic findings. The histopathologic differential diagnosis is broad, requiring knowledge of the possible diagnoses at each specific anatomic site, and a careful assessment to exclude other inflammatory and neoplastic disorders. An immunohistochemistry panel including CD163, CD1a, langerin, S100, Factor XIIla, OCT2, and $B R A F$ V600E can provide definitive diagnosis in LCH and RDD, whereas ECD requires classic clinical features as well as confirmation of an activating MAPK pathway mutation by genetic studies.
\end{abstract}

J Natl Compr Canc Netw 2021;19(11):1305-1311 doi: $10.6004 /$ jnccn.2021.7098

${ }^{1}$ Division of Hematopathology, Department of Laboratory Medicine and Pathology, Mayo Clinic, Rochester, Minnesota.
Within the past decade, discoveries of pathogenic mutations in the MAPK (RAS-RAF-MEK-ERK) pathway and PI3K-AKT pathway in histiocytic neoplasms have transformed our ability to diagnose, classify, and treat these disorders. ${ }^{1-4}$ However, the first step in confirming a pathologic diagnosis in these patients remains a great challenge. These disorders, including Langerhans cell histiocytosis (LCH), Erdheim-Chester disease (ECD), and RosaiDorfman disease (RDD), show a variety of clinical presentations that present to many different specialty areas, including dermatology, neurology, hematology, endocrinology, pulmonology, cardiology, and rheumatology. ${ }^{3}$ Localized and systemic symptoms are often nonspecific and can overlap with other common conditions. The diagnosis requires multidisciplinary integration of the entire picture from the clinical, radiology, laboratory, and pathology perspectives.

\section{Clinical Presentation}

The presenting signs and symptoms of histiocytic neoplasms are diverse, and careful attention to the clinical presentation is key to both diagnosis and accurate classification. Patients presenting with clinical manifestations such as fatigue, bone pain, perinephric infiltrates, diabetes insipidus, and cerebellar syndromes alert the clinician to a systemic disease process. ${ }^{3}$ When histiocytic neoplasms present with these systemic symptoms, they will often fall into the "L group" as classified by the Histiocyte Society (LCH, ECD, and mixed histiocytosis). ${ }^{5}$ In this setting, the role of the pathologist is to interpret the biopsy in this context and exclude other systemic conditions. In other cases, the pathologist is first presented with incidental histiocytic infiltrates in a cutaneous or lymph node biopsy. That situation requires the pathologist to characterize the histiocytic infiltrate and alert the clinician to the possibility of a systemic histiocytic neoplasm. The histiocytic process may ultimately be determined to be a manifestation of a systemic L group histiocytosis or may fall into the "C group" (histiocytosis limited to the skin) or the "R group" (nodal and extranodal RDD).

\section{Choosing the Biopsy Site}

Even in the setting of a clinically suspected histiocytic neoplasm, pathologic confirmation is difficult given the 
nonspecific histologic findings. Retrospective expert review of prior biopsies taken before a histiocytic neoplasm was suspected may yield a definitive diagnosis. It is not uncommon, however, to require multiple biopsies to provide adequate tissue for diagnosis. ${ }^{3}$ Discussion between the clinician, radiologist, and pathologist is important to identify the biopsy site that is most accessible, appears to represent active disease on imaging studies, and will provide a tissue specimen with adequate histology and cellularity. When a bone lesion is selected for biopsy, a portion of the tissue should be processed without decalcification or decalcified with an EDTA-based method that does not inhibit molecular genetic studies.

\section{Histopathology}

Histiocytic neoplasms often are difficult to diagnose because of histologic features that overlap with reactive fibroinflammatory infiltrates. In the 3 major types, $\mathrm{LCH}, \mathrm{RDD}$, and ECD, the histiocyte morphology is variable and bland and may be obscured by fibrosis and intermixed reactive inflammation. The histiocytes in each type have a distinct phenotypic profile. A useful immunohistochemistry (IHC) panel to characterize the histiocytes includes CD68 or CD163, S100, CD1a, langerin, Factor XIIIa, and cyclin D1 (Table 1). Additional useful markers include OCT2 and BRAF V600E (antibody clone VE1). Although a more limited IHC panel may be adequate as guided by morphologic features, application of the full panel can help identify cases of mixed histiocytoses in which $\mathrm{LCH}, \mathrm{ECD}$, and RDD components are present within the same biopsy or involving different sites in the same patient. ${ }^{6,7}$

\begin{tabular}{|lccc|}
\hline \multicolumn{2}{|l|}{ Table 1. IHC of Histiocytic Diseases } & & \\
\hline IHC Marker & LCH & RDD & ECD \\
\hline CD68 (cytoplasmic) & $+^{\mathrm{a}}$ & + & + \\
\hline CD163 (cytoplasmic) & $-^{\mathrm{b}}$ & $+^{\mathrm{c}}$ & + \\
\hline Cyclin D1 (nuclear \pm cytoplasmic) & + & + & \pm \\
\hline S100 (nuclear and cytoplasmic) & + & + & $\pm^{\mathrm{d}}$ \\
\hline OCT2 (nuclear) & $-{ }^{\mathrm{b}}$ & + & - \\
\hline Factor XIIla (nuclear and cytoplasmic) & - & $\pm^{\mathrm{e}}$ & + \\
\hline CD1a/Langerin (membrane/cytoplasmic) & + & - & $\times$ \\
\hline BRAF V600E (cytoplasmic) & $+^{\mathrm{f}}$ & - & $+^{\mathrm{f}}$ \\
\hline
\end{tabular}

Abbreviations: ECD, Erdheim-Chester disease; IHC, immunohistochemistry; LCH, Langerhans cell histiocytosis; RDD, Rosai-Dorfman disease.

a In LCH, CD68 shows a paranuclear dot-like staining.

bOCT2 and CD163 can be expressed in a minority of LCH cases (5\%-10\%).

${ }^{\circ} \mathrm{CD} 163$ is negative in a minority of RDD cases $(\sim 10 \%)$.

e Factor XIIla is expressed in $30 \%$ of RDD cases.

${ }^{d} \mathrm{~S} 100$ is expressed in $20 \%-30 \%$ of ECD cases.

${ }^{f} B R A F$ V600E mutation is present in $50 \%-60 \%$ of $L C H$ and ECD cases.

\section{Langerhans Cell Histiocytosis}

The pathologic diagnosis of LCH relies on identification of abnormal collections of cells with a Langerhans cell phenotype. The cells of LCH show characteristic morphology with reniform nuclei, frequent longitudinal nuclear grooves, pale chromatin, and abundant eosinophilic cytoplasm. ${ }^{8,9}$ A reactive inflammatory infiltrate accompanies the neoplastic cells and includes eosinophils, which may be numerous; small lymphocytes; and macrophages (Figure 1). Multinucleated giant cells may be present. In certain anatomic sites, in particular bone and pulmonary lesions, there is evolution over time from cellular infiltrates to "burned-out" lesions with patchy cellularity, increased fibrosis, and foamy histiocytes. ${ }^{5,10}$ Within skin lesions, the neoplastic cells of LCH are distinguished from reactive Langerhans cell populations by their enlarged nuclei, sheet-like collections, and lack of dendritic processes. In lymph nodes, LCH involves and expands the sinuses. This localization within the sinuses is a critical distinction from the reactive Langerhans cells that are present in the paracortex of lymph nodes with benign dermatopathic change. ${ }^{11}$

The phenotype of $\mathrm{LCH}$ is not distinct from that of benign Langerhans cells, with expression of CD68, S100, $\mathrm{CDla}$, and langerin. They are commonly negative for monocyte/macrophage markers CD14, CD163, and OCT2. ${ }^{12}$ In contrast to benign Langerhans cells that may show weak and patchy cyclin D1 expression, the neoplastic cells of LCH show strong and diffuse expression of cyclin D1 as a result of the activation of the MAPK pathway, ${ }^{13,14}$ and this feature may be helpful in diagnosis.

Given a diagnostic morphology and phenotype, identification of a mutation in the MAPK pathway is not required to establish a diagnosis of LCH. However, patients who require treatment will benefit from identification of pathogenic mutations that may allow targeted therapy.

\section{Rosai-Dorfman Disease}

Classic (nodal) RDD shows a similar morphologic pattern to $\mathrm{LCH}$, with localization of the abnormal histiocytes within the lymph node sinuses. The enlargement of the lymph node is due to both the distension of the sinuses by histiocytes and hyperplasia of lymphocytes and plasma cells within the lymph node parenchyma. Confirmation of the RDD phenotype is necessary to distinguish RDD from nonspecific reactive histiocytes within the sinuses (sometimes called "sinus histiocytosis").

Compared with classic (nodal) RDD, extranodal RDD shows a more nonspecific histologic pattern, making it more critical to identify the characteristic RDD histiocyte. The RDD histiocyte displays recognizable cytologic features with a large round-to-oval nucleus, pale chromatin, a pinpoint nucleolus, and voluminous cytoplasm. A common feature of these histiocytes is the presence of 

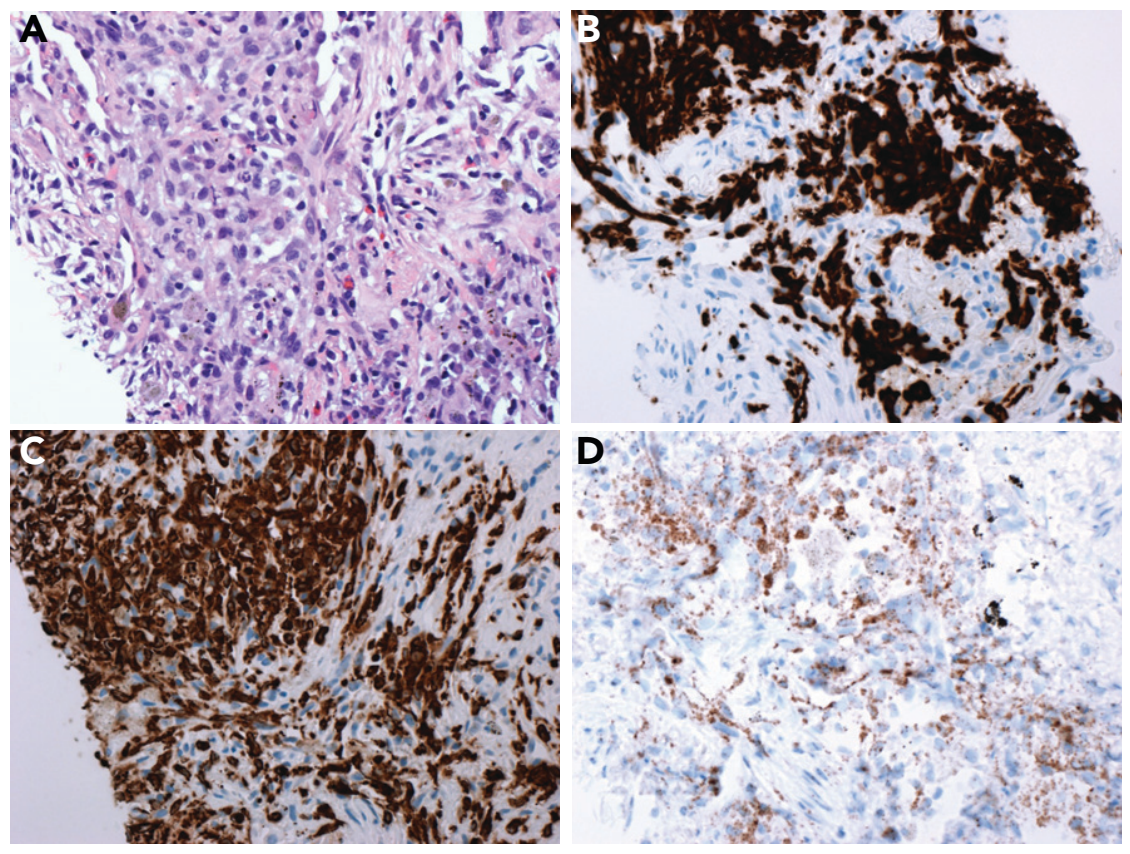

Figure 1. Lung biopsy involved by Langerhans cell histiocytosis. (A) Hematoxylin-eosin stain showing mixed inflammation, including clusters of Langerhans cells, small lymphocytes, and rare eosinophils in a fibrotic background. The Langerhans cells are positive for (B) CD1a, (C) langerin, and (D) BRAF V600E by immunohistochemistry (original magnification $\times 400$ for A-D).

emperipolesis, with intact lymphocytes or plasma cells engulfed within the cytoplasm. It should be noted, however, that emperipolesis is not specific for RDD, and may be found in other histiocytic neoplasms as well as reactive histiocytic infiltrates. ${ }^{5,15}$ The RDD histiocytes are often singly distributed in a reactive inflammatory background that includes lymphoid aggregates, numerous plasma cells, and fibrosis. Given these features, the RDD histiocytes can be difficult to identify, and entities such as malignant lymphoma, IgG4-related disease, infectious processes, and autoimmune disease are diagnostic considerations. The IHC panel used should therefore include markers for RDD histiocytes as well as those necessary to exclude these other entities.

RDD shows expression of macrophage markers CD68 and CD163, as well the monocyte marker OCT2. ${ }^{12}$ In addition, $\mathrm{S} 100$ is positive in the RDD histiocytes, whereas CDla and langerin are negative. The diffuse cytoplasmic staining for CD163 and S100 is useful both to identify the RDD histiocytes and to highlight the presence of emperipolesis. However, given that CD163 may be negative in up to $10 \%$ of RDD cases and S100 expression may also be weak to negative, CD68 and OCT2 can be used as alternative markers to confirm the macrophage/monocyte phenotype. ${ }^{12}$ When interpreting the OCT2 immunostain, it is important to remember that OCT2 is expressed by B cells and plasma cells as well as RDD histiocytes. As in LCH, cyclin D1 is overexpressed in RDD. ${ }^{16}$ In cases with only rare RDD cells in an inflammatory background, identification of histiocytes expressing both cyclin D1 and OCT2 is helpful (Figure 2). The RDD histiocytes are often located at the periphery of reactive lymphoid follicles within the infiltrate.

Classic (nodal) RDD and cutaneous RDD are often self-limited and do not progress to systemic disease requiring treatment, and in these cases identification of a MAPK pathway mutation is not required for diagnosis. However, it should be noted that MAPK pathway mutations have been identified in approximately one-third of patients with RDD, suggesting that a subset represent clonal neoplasms. ${ }^{17}$ RDD may show a similar presentation to ECD, with involvement of perinephric soft tissue and bone lesions. When RDD presents as disseminated disease, analysis for MAPK pathway mutations is recommended. More rarely, RDD may be seen in hereditary diseases including Faisalabad syndrome or FAS deficiency, in which case germline genetic testing may be indicated.

\section{Erdheim-Chester Disease}

The histopathology of ECD falls under the family of (juvenile) xanthogranuloma lesions. This pathologic diagnosis can be the most challenging of the histiocytic neoplasms, because the morphology shows nonspecific lymphohistiocytic infiltrates located exclusively in extranodal sites. ${ }^{18}$ The classic histologic picture shows an infiltrate of abundant foamy histiocytes accompanied by Touton-type giant cells. However, the foamy histiocytes show minimal cytologic atypia, making ECD indistinguishable from benign xanthogranulomatous infiltrates. Furthermore, 

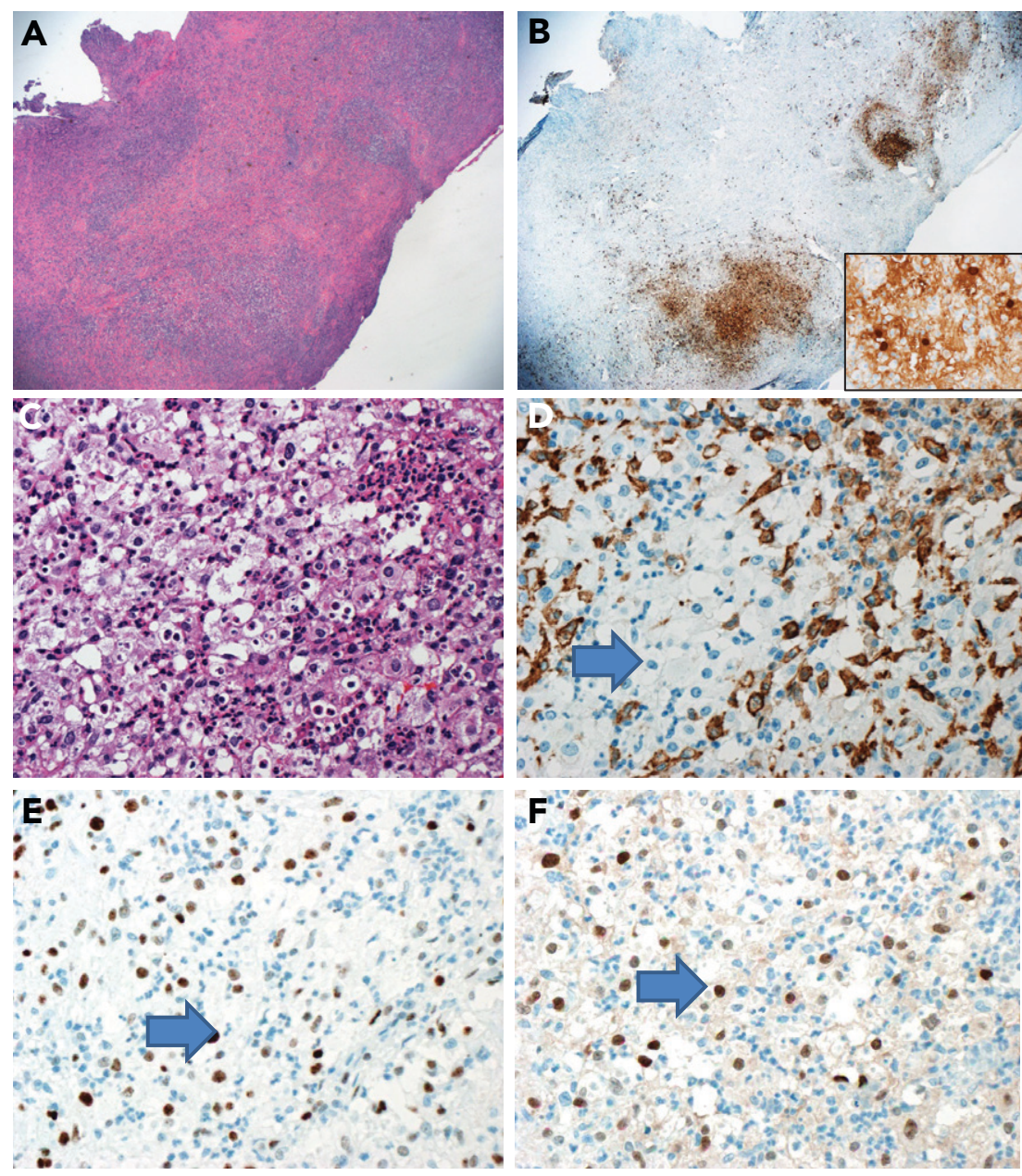

Figure 2. Paraspinal soft tissue involved by Rosai-Dorfman disease. (A) Hematoxylin-eosin stain showing nodular lymphoplasmacytic infiltrates with surrounding dense fibrosis. (B) Clusters of S100-positive histiocytes are present. (C) Higher magnification shows the histiocytes with round, vesicular nuclei, and abundant cytoplasm with emperipolesis, in a background of plasma cells and neutrophils. (D) CD163 is negative in the RDD histiocytes in $\sim 10 \%$ of RDD, as in this case (arrow). The coexpression of (E) OCT2 and (F) cyclin D1 identifies the RDD histiocytes (arrows) (original magnification $\times 20$ for $A$, $B$; original magnification $\times 400$ for $B$ inset and $C-F)$.

the classic histologic picture is frequently lacking, and instead the infiltrates can show a variable composition of different morphologic types of histiocytes ${ }^{18}$ (Figure 3 ). The morphologic variants have been described as having scalloped, vacuolated, xanthomatized, spindle cell, or oncocytic cytoplasmic features. ${ }^{15,19}$ It has been suggested that these variants represent a continuum along a maturation pathway that may change over the temporal evolution of the lesion, and also may be influenced by the nature of the host response..$^{20}$

In addition to nonspecific histology, the IHC phenotype does not always distinguish ECD from other inflammatory infiltrates. Macrophage markers including CD163 and CD68 are expressed, whereas markers of Langerhans cell differentiation (CDla, langerin) are absent. ${ }^{18} \mathrm{~S} 100$ is negative or weakly expressed in a minority of cases. Although strong nuclear and cytoplasmic expression of
Factor XIIIa is characteristic and supportive of a diagnosis of ECD, this marker is also positive in localized forms of xanthogranuloma, ${ }^{19}$ may be expressed in RDD, and is variably expressed in reactive stromal cells. ${ }^{12}$ In contrast to LCH and RDD, cyclin D1 has not been established as a marker that is useful in distinguishing ECD histiocytes from their reactive counterparts. Therefore, a diagnosis of ECD often cannot be established based on histologic features alone.

The most frequent sites of involvement in ECD include the long bones, retroperitoneum, lung, orbits, skin, and central nervous system (CNS). When a bone lesion is targeted for biopsy, advanced planning is necessary to process multiple cores without decalcification, as this may interfere with IHC for BRAF V600E as well as molecular genetic testing. In the bone, ECD often shows fibrosis and scant cellularity, which also limits the 

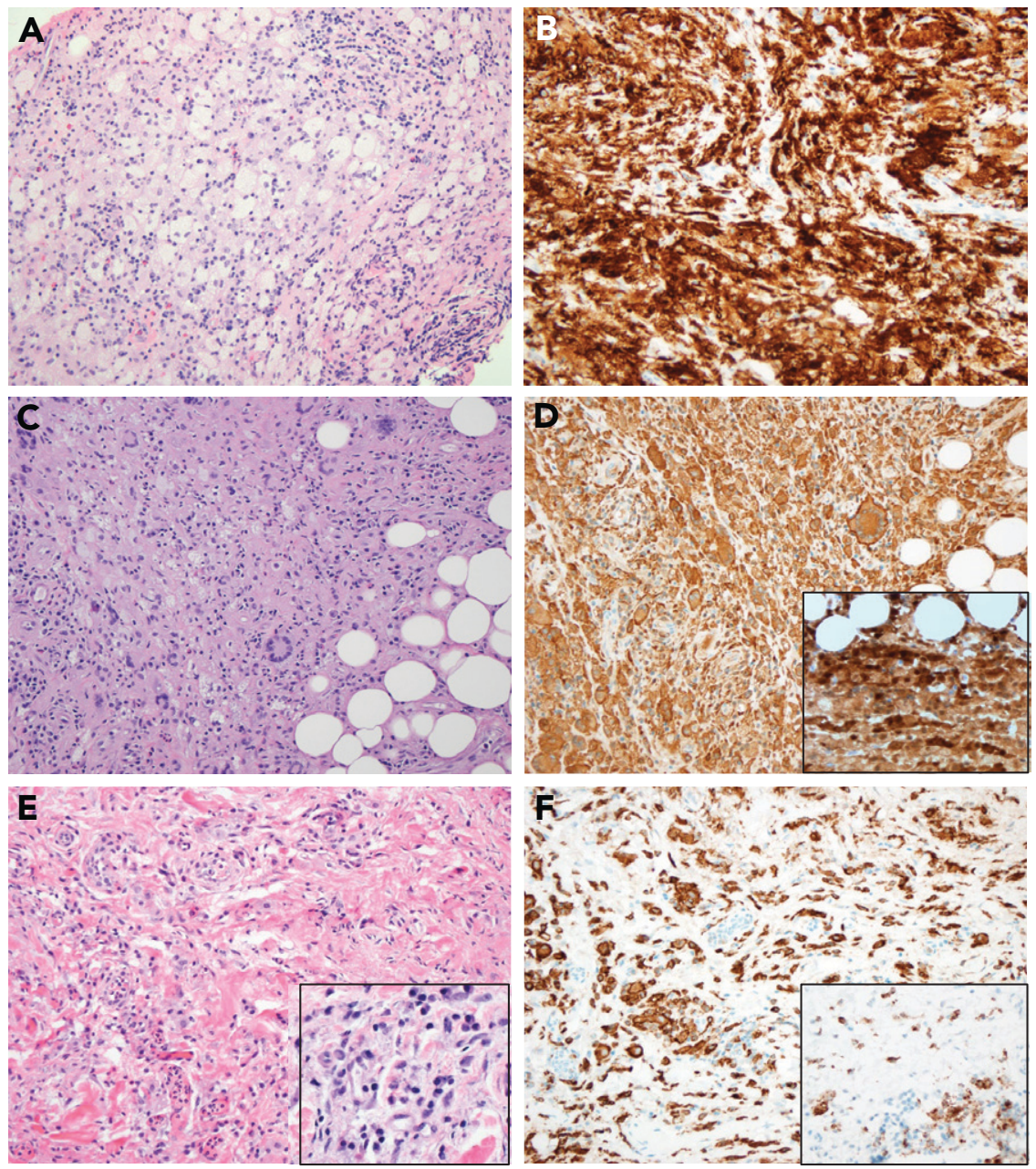

Figure 3. Variable histopathology in Erdheim-Chester disease. (A, B) Classic xanthogranulomatous histopathology in the orbit with abundant foamy histiocytes and scattered lymphoplasmacytic infiltrate. Factor XIlla shows strong nuclear and cytoplasmic expression throughout the infiltrate. A BRAF V600E mutation was confirmed by PCR. (C, D) Mesenteric infiltrates include a mixture of histiocytes with vacuolated cytoplasm and Touton giant cells. The CD163 positive histiocytes are also strongly positive for Factor XIIla (inset). An activating KRAS mutation was found on next-generation sequencing studies. (E, F) In this retroperitoneal biopsy, the histiocytes show spindled morphology and are sparsely distributed in a hyalinized fibrotic background. CD163 highlights the histiocytes that are BRAFV600E mutated by immunohistochemistry (inset) (original magnification $\times 200$ for A-F; original magnification $\times 400$ for insets).

success of genetic studies. In this location, other processes such as osteomyelitis, fibrous dysplasia, or giant cell-rich lesions including giant cell tumor of bone and pigmented villonodular synovitis may show histologic overlap with ECD. The differential diagnosis also includes LCH and more rarely RDD, which can be distinguished by IHC studies.

The retroperitoneum is a frequent site of ECD involvement, in the form of perinephric ("hairy kidney") or periaortic infiltrates ("coated aorta"). Occasionally, the mesentery or peritoneum may also be involved. ${ }^{21}$ The differential diagnosis is broad at these locations, to include xanthogranulomatous pyelonephritis, fat necrosis, IgG4-related disease, malignant lymphoma (follicular lymphoma characteristically is accompanied by fibrosis at this site), liposarcoma, infection (especially histoplasmosis), and idiopathic retroperitoneal fibrosis/sclerosing mesenteritis.

In orbital xanthogranulomatous infiltrates, the differential diagnosis includes several benign entities as well as ECD. ${ }^{18,22}$ The location of the infiltrates is an important distinction, with ECD involving the intraconal orbital soft tissue, which can result in exophthalmos. In contrast, benign xanthogranulomatous lesions are usually limited to the preseptal anterior orbit. These clinicopathologic entities include adult-onset asthma periorbital xanthogranuloma (AAPOX), necrobiotic xanthogranuloma (NXG), and xanthelasma. The clinical history is important to identify the association of asthma with AAPOX, monoclonal serum immunoglobulins with NXG, and cholesterol disorders 
with xanthelasma. The orbit and lacrimal gland may also be sites of involvement of IgG4-related disease and extranodal marginal zone lymphoma.

Cutaneous xanthogranulomatous histiocytic diseases display a diverse clinicopathologic spectrum, with most showing a self-limited course without systemic disease These have been divided into those that are (1) limited to the skin (benign cephalic histiocytosis, juvenile xanthogranuloma [JXG], generalized eruptive histiocytosis, adult xanthogranuloma [AXG], progressive nodular histiocytosis, solitary reticulohistiocytoma, indeterminate histiocytosis); (2) cutaneous lesions with a major systemic component (xanthoma disseminatum, multicentric reticulohistiocytoma); and (3) systemic histiocytosis (ECD) ${ }^{5,20}$ Histologic differences have been described for each of these entities; for example, JXG/AXG contains many xanthomatized (foamy) histiocytes and Touton giant cells, whereas solitary or multicentric reticulohistiocytoma is composed of mostly of oncocytic histiocytes and multinucleated giant cells with glassy cytoplasm. In progressive nodular histiocytosis, spindled histocytes predominate. However, histologic overlap prevents distinction based on pathology alone, and familiarity with the specific clinical presentation of each of these disorders is necessary for classification. ${ }^{23,24} \mathrm{In}$ some cases, xanthogranulomatous skin lesions will be the first presentation of ECD, prompting a careful evaluation of the patient to identify systemic disease. If a systemic process is confirmed or suspected, skin lesions provide an accessible biopsy site for molecular genetic testing.

ECD involvement of the brain is particularly challenging for diagnosis. Brain involvement with ECD often shows a subtle parenchymal infiltrate by single histiocytes with nonlipidized cytoplasm, ${ }^{18}$ and in other cases presents with mass lesions of the dura or parenchyma. ${ }^{15}$ The background lacks the typical fibrotic stroma and may show reactive astrocytic proliferation with prominent Rosenthal formation, obscuring the diagnostic infiltrate. When ECD involving the brain is suspected, the confirmation of diagnosis may be most easily obtained by identification and biopsy of an alternative site of involvement, such as skin, retroperitoneum, or bone. ${ }^{25}$

In contrast to LCH and RDD, identification of a pathogenic mutation in the MAPK or PI3K pathways is critical to confirm a pathologic diagnosis of ECD. The specific mutation found is also essential to direct therapeutic options.

\section{Gene Mutation Analysis}

Activating mutations involving the genes of the MAPK and (PI3K)-AKT pathways have been found in LCH,

\section{Table 2. Advantages and Limitations of Various Molecular Test Platforms}

\begin{tabular}{|c|c|c|c|}
\hline Test Platform & Advantages & Limitations & $\begin{array}{l}\text { Utilization in Histiocytic } \\
\text { Neoplasm Workup }\end{array}$ \\
\hline AS-PCR & $\begin{array}{l}\text { - Highly sensitive }\left(10^{-4} \text { to } 10^{-6}\right) \\
\text { - Inexpensive } \\
\text { - Faster TAT }\end{array}$ & Only examines specific mutation targets & BRAF V600 mutation detection \\
\hline ddPCR & $\begin{array}{l}\text { - Highly sensitive }\left(10^{-3} \text { to } 10^{-6}\right) \\
\text { - Inexpensive } \\
\text { - Faster TAT }\end{array}$ & Only examines specific mutation targets & BRAF V600 mutation detection \\
\hline Targeted NGS panel & $\begin{array}{l}\text { Examines multiple gene targets } \\
\text { simultaneously }\end{array}$ & $\begin{array}{l}\text { - Lower sensitivity than AS-PCR and ddPCR } \\
\left(3 \%-5 \%{ }^{a}\right) \\
\text { - Average depth of coverage } \times 500-1,000 \\
\text { - More expensive and longer TAT given increased } \\
\text { technical and analytical complexities }\end{array}$ & $\begin{array}{l}\text { Targeted sequencing of } \\
\text { commonly mutated genes, } \\
\text { including ARAF, BRAF, KRAS, } \\
\text { NRAS, MAP2K1, PI3KCA, and } \\
\text { CSF1R }\end{array}$ \\
\hline WES & $\begin{array}{l}\text { Examines the coding regions } \\
\text { (exome) of the genome }\end{array}$ & $\begin{array}{l}\text { - Lower sensitivity than targeted NGS panel } \\
(10 \%-30 \%) \\
\text { - Average depth of coverage } \times 30-100 \\
\text { - Costly and long TAT due to significantly } \\
\text { increased technical and analytical complexities }\end{array}$ & $\begin{array}{l}\text { Not recommended given } \\
\text { limited advantage over target } \\
\text { NGS panel }\end{array}$ \\
\hline RNA sequencing & $\begin{array}{l}\text { - Examines the RNA transcriptome } \\
\text { - Has the ability to assess gene } \\
\text { expression levels, novel } \\
\text { transcripts, splicing isoforms, } \\
\text { nucleotide variations }{ }^{b}\end{array}$ & $\begin{array}{l}\text { - Required read depth varies depending on the } \\
\text { goals of the study (most require 5-200 million } \\
\text { reads per sample) } \\
\text { - Sensitivity impacted by RNA transcript } \\
\text { expression level and the type of RNA } \\
\text { information sought } \\
\text { - RNA template labile, test may be compromised } \\
\text { in FFPE samples with highly degraded RNA, } \\
\text { may need fresh frozen sample or fresh sample } \\
\text { in RNA preservatives } \\
\text { - Costly and long TAT due to significantly } \\
\text { increased technical and analytical complexities }\end{array}$ & $\begin{array}{l}\text { Identification of gene fusions in } \\
B R A F, A L K, N T R K 1 \text {, etc, if no } \\
\text { mutations detected }\end{array}$ \\
\hline
\end{tabular}

Abbreviations: AS-PCR, allele-specific PCR; ddPCR, droplet digital PCR; FFPE, formalin-fixed paraffin-embedded; NGS, next-generation sequencing; TAT, turnaround time; WES, whole-exome sequencing.

${ }^{a}$ Analytical sensitivity can be greatly improved with error-corrected NGS.

${ }^{b}$ The extent of genetic aberrancies assessable by each clinical assay is dependent on the bioinformatic tools, test design, and test validation. 
RDD, and ECD. As a first step in identifying a mutation, IHC using the VE1 clone for BRAF V600E can rapidly identify this mutant protein that is present in approximately half of LCH and ECD cases. Careful optimization of the IHC protocol is important to maximize the sensitivity and minimize nonspecific staining. In the first author's experience (K.L. Rech), a protocol that uses a tyramide-based amplification step is necessary to detect the low levels of mutant protein expression in histiocytic neoplasms. In the setting of a negative or equivocal BRAF V600E IHC stain or if the IHC stain is not available, confirmation by allele-specific PCR or digital PCR for $B R A F$ V600 mutation(s) can be performed (Table 2). However, as an alternative next step, next-generation sequencing (NGS) testing using a targeted gene panel that at a minimum includes ARAF, BRAF, KRAS, NRAS, MAP2K1, $P I 3 K C A$, and CSF1R can provide a more comprehensive analysis. Because the variant allele frequency of pathogenic mutations is often very low in $\mathrm{ECD},{ }^{26}$ it is not uncommon to require additional tissue biopsy and repeat NGS studies and/or a highly sensitive molecular test before the pathogenic mutation is identified. If no mutation is found, additional testing by RNA sequencing would be required to identify pathogenic gene fusions of BRAF, $A L K$, or NTRK1. As whole-exome sequencing (WES) provides limited additional value beyond a well-designed targeted NGS panel covering recurrent mutations of histiocytic neoplasms, routine WES testing is not currently recommended in the clinical setting.

\section{Conclusions}

The diagnosis of histiocytic neoplasms remains a great challenge. Increased awareness of the clinical, radiologic, and histologic features among physicians will foster the multidisciplinary collaboration that is the key to diagnosis. Identification of pathogenic mutations associated with these diseases enhances our diagnostic ability and shows great promise for a personalized therapeutic approach for this group of patients.

Submitted June 14, 2021; final revision received September 24, 2021 accepted for publication September 28, 2021

Disclosures: The authors have disclosed not having any financial interests, arrangements, or affiliations with the manufacturers of any products discussed in this article or their competitors.

Correspondence: Karen L. Rech, MD, Division of Hematopathology, Department of Laboratory Medicine and Pathology, Mayo Clinic, 200 First Street SW, Rochester, MN 55905. Email: rech.karen@mayo.edu

\section{References}

1. Goyal G, Ravindran A, Young JR, et al. Clinicopathological features, treatment approaches, and outcomes in Rosai-Dorfman disease. Haematologica 2020; 105:348-357.

2. Goyal G, Heaney ML, Collin M, et al. Erdheim-Chester disease: consensus recommendations for evaluation, diagnosis, and treatment in the molecular era. Blood 2020;135:1929-1945.

3. Goyal G, Young JR, Koster MJ, et al. The Mayo Clinic Histiocytosis Working Group consensus statement for the diagnosis and evaluation of adult patients with histiocytic neoplasms: Erdheim-Chester disease, Langerhans cell histiocytosis, and Rosai-Dorfman disease. Mayo Clin Proc 2019;94:2054-2071.

4. Diamond $\mathrm{EL}$, Durham BH, Haroche J, et al. Diverse and targetable kinase alterations drive histiocytic neoplasms. Cancer Discov 2016;6:154-165.

5. Emile JF, Abla O, Fraitag S, et al. Revised classification of histiocytoses and neoplasms of the macrophage-dendritic cell lineages. Blood 2016;127:2672-2681.

6. Bonometti A; for Associazione Italiana Ricerca Istiocitosi AIRI ONLUS. The triptych of mixed histiocytosis: a systematic review of 105 cases and proposed clinical classification. Leuk Lymphoma 2021;62:32-44.

7. Hervier B, Haroche J, Arnaud L, et al. Association of both Langerhans cell histiocytosis and Erdheim-Chester disease linked to the BRAFV600E mutation. Blood 2014;124:1119-1126.

8. Roden AC, Hu X, Kip S, et al. BRAF V600E expression in Langerhans cell histiocytosis: clinical and immunohistochemical study on 25 pulmonary and 54 extrapulmonary cases. Am J Surg Pathol 2014;38:548-551.

9. Satpathy AT, Kc W, Albring JC, et al. Zbtb46 expression distinguishes classical dendritic cells and their committed progenitors from other immune lineages. J Exp Med 2012;209:1135-1152.

10. Roden AC, Yi ES. Pulmonary Langerhans cell histiocytosis: an update from the pathologists' perspective. Arch Pathol Lab Med 2016;140:230-240.

11. Ravindran A, Goyal G, Failing JJ, et al. Florid dermatopathic lymphadenopathy-a morphological mimic of Langerhans cell histiocytosis. Clin Case Rep 2018;6:1637-1638.

12. Ravindran A, Goyal G, Go RS, et al. Rosai-Dorfman disease displays a unique monocyte-macrophage phenotype characterized by expression of OCT2. Am J Surg Pathol 2021;45:35-44
13. Chatterjee D, Vishwajeet V, Saikia UN, et al. CyclinD1 is useful to differentiate Langerhans cell histiocytosis from reactive Langerhans cells. Am J Dermatopathol 2019;41:188-192.

14. Shanmugam V, Craig JW, Hornick JL, et al. Cyclin D1 is expressed in neoplastic cells of Langerhans cell histiocytosis but not reactive Langerhans cell proliferations. Am J Surg Pathol 2017;41:1390-1396.

15. Picarsic J, Pysher $\mathrm{T}$, Zhou $\mathrm{H}$, et al. BRAF V600E mutation in juvenile xanthogranuloma family neoplasms of the central nervous system (CNSJXG): a revised diagnostic algorithm to include pediatric Erdheim-Chester disease. Acta Neuropathol Commun 2019;7:168.

16. Baraban E, Sadigh S, Rosenbaum J, et al. Cyclin D1 expression and novel mutational findings in Rosai-Dorfman disease. Br J Haematol 2019;186:837-844.

17. Abla O, Jacobsen E, Picarsic J, et al. Consensus recommendations for the diagnosis and clinical management of Rosai-Dorfman-Destombes disease. Blood 2018;131:2877-2890.

18. Ozkaya N, Rosenblum MK, Durham BH, et al. The histopathology of Erdheim-Chester disease: a comprehensive review of a molecularly characterized cohort. Mod Pathol 2018;31:581-597.

19. Zelger BW, Sidoroff A, Orchard G, et al. Non-Langerhans cell histiocytoses. A new unifying concept. Am J Dermatopathol 1996;18:490-504.

20. Weitzman S, Jaffe R. Uncommon histiocytic disorders: the non-Langerhans cell histiocytoses. Pediatr Blood Cancer 2005;45:256-264.

21. Cohen-Aubart F, Ungureanu I, Razanamahery J, et al. Peritoneal or mesenteric tumours revealing histiocytosis. BMJ Open Gastroenterol 2021;8:8.

22. Kerstetter J, Wang J. Adult orbital xanthogranulomatous disease: a review with emphasis on etiology, systemic associations, diagnostic tools, and treatment. Dermatol Clin 2015;33:457-463.

23. Kobic A, Shah KK, Schmitt AR, et al. Erdheim-Chester disease: expanding the spectrum of cutaneous manifestations. Br J Dermatol 2020;182:405-409.

24. Bonomett A, Berti E; for Associazione Italiana Ricerca Istiocitosi ONLUS. Reticulohistiocytoses: a revision of the full spectrum. J Eur Acad Dermatol Venereol 2020;34:1684-1694.

25. Budhram A, Rech KL, Peikert JM, et al. Teaching neuroimages: brain and skin involvement in Erdheim-Chester disease. Neurology 2021;96:e1590-1592.

26. Melloul S, Hélias-Rodzewicz Z, Cohen-Aubart F, et al. Highly sensitive methods are required to detect mutations in histiocytoses. Haematologica 2019;104:e97-99. 